OPEN ACCESS

Edited by:

Xin Di,

New Jersey Institute of Technology

United States

Reviewed by:

Tiffany A. Kolesar,

University of Manitoba, Canada

Dapeng Liu,

Johns Hopkins University,

United States

*Correspondence:

Ya-Chung Tian

dryctian@cgmh.org.tw

Changwei W. Wu

sleepbrain@tmu.edu.tw

tThese authors have contributed equally to this work and share senior authorship

Specialty section:

This article was submitted to

Nephrology,

a section of the journal

Frontiers in Medicine

Received: 01 July 2021 Accepted: 27 October 2021

Published: 24 November 2021

Citation:

Chang T-Y, Wu H-H, Li Y-J, Liu H-L,

Yeh $C-H$, Jian $H-S$, Huang $K-L$,

Lee T-H, Tian Y-C and Wu CW (2021)

Changes of Brain Functional

Connectivity in End-Stage Renal

Disease Patients Receiving Peritoneal

Dialysis Without Cognitive Decline.

Front. Med. 8:734410.

doi: 10.3389/fmed.2021.734410

\section{Changes of Brain Functional Connectivity in End-Stage Renal Disease Patients Receiving Peritoneal Dialysis Without Cognitive Decline}

\author{
Ting-Yu Chang ${ }^{1}$, Hsin-Hsu Wu ${ }^{2,3}$, Yi-Jung Li ${ }^{2,3}$, Ho-Ling Liu ${ }^{4}$, Chih-Hua Yeh ${ }^{5}$, \\ Hui-Shan Jian ${ }^{5}$, Kuo-Lun Huang ${ }^{1}$, Tsong-Hai Lee ${ }^{1}$, Ya-Chung Tian ${ }^{2,3 *+}$ and \\ Changwei W. $W u^{6,7 * t}$
}

${ }^{1}$ Department of Neurology, Stroke Section, Chang Gung Memorial Hospital, Linkou Medical Center and College of Medicine, Chang Gung University, Taoyuan, Taiwan, ${ }^{2}$ Graduate Institute of Clinical Medical Sciences, Chang Gung University, Taoyuan, Taiwan, ${ }^{3}$ Kidney Research Center, Department of Nephrology, Chang Gung Memorial Hospital, Linkou Medical Center and College of Medicine, Chang Gung University, Taoyuan, Taiwan, ${ }^{4}$ Department of Imaging Physics, The University of Texas M.D. Anderson Cancer Center, Houston, TX, United States, ${ }^{5}$ Department of Medical Imaging and Intervention, Chang Gung Memorial Hospital, Taoyuan City, Taiwan, ${ }^{6}$ Graduate Institute of Mind, Brain and Consciousness, Taipei Medical University, Taipei, Taiwan, ${ }^{7}$ Brain and Consciousness Research Center, Shuang-Ho Hospital-Taipei Medical University, New Taipei, Taiwan

Background: Functional connectivity detected by resting-state functional MRI (R-fMRI) helps to discover the subtle changes in brain activities. Patients with end-stage renal disease (ESRD) on hemodialysis (HD) have impaired brain networks. However, the functional changes of brain networks in patients with ESRD undergoing peritoneal dialysis (PD) have not been fully delineated, especially among those with preserved cognitive function. Therefore, it is worth knowing about the brain functional connectivity in patients with PD by using R-fMRI.

Methods: This case-control study prospectively enrolled 19 patients with ESRD receiving PD and 24 age- and sex- matched controls. All participants without a history of cognitive decline received mini-mental status examination (MMSE) and brain 3-T R-fMRI. Comprehensive R-fMRI analyses included graph analysis for connectivity and seed-based correlation networks. Independent $t$-tests were used for comparing the graph parameters and connectivity networks between patients with PD and controls.

Results: All subjects were cognitively intact (MMSE > 24). Whole-brain connectivity by graph analysis revealed significant differences between the two groups with decreased global efficiency ( $\left.E_{g l o b}, p<0.05\right)$, increased betweenness centrality $(B C)(p<0.01)$, and increased characteristic path length $(L, p<0.01)$ in patients with PD. The functional connections of the default-mode network (DMN), sensorimotor network (SMN), salience network $(\mathrm{SN})$, and hippocampal network $(\mathrm{HN})$ were impaired in patients with PD. Meanwhile, in DMN and SN, elevated connectivity was observed in certain brain regions of patients with PD. 
Conclusion: Patients with ESRD receiving PD had specific disruptions in functional connectivity. In graph analysis, $E_{\text {glob }}, B C$, and $L$ showed significant connectivity changes compared to the controls. DMN and $\mathrm{SN}$ had the most prominent alterations among the observed networks, with both decreased and increased connectivity regions. Our study confirmed that significant changes in cerebral connections existed in cognitively intact patients with $P D$.

Keywords: end-stage renal disease, peritoneal dialysis, resting-state functional MRI, functional connectivity, graph theory, default-mode network, cognitive function

\section{INTRODUCTION}

End-stage renal disease (ESRD) causes a severe burden on global public health and has a poor prognosis. According to a recent report (1), the Taiwanese population has the highest prevalence of ESRD with dialysis therapy in the world. Asides from systemic complications, neurological manifestations are common in the ESRD population. Studies over the past decade showed that when compared with healthy individuals, patients with ESRD had a higher incidence of stroke (2), cognitive impairment, and dementia, regardless of their renal replacement modalities $(3,4)$.

The underlying mechanism of higher incidence of cerebral diseases in patients with ESRD, the so-called "kidney-brain axis," is complicated. Based on the similarity of anatomical and vasoregulatory features of the brain and kidneys, vasculopathy of cerebral and glomerular small vessels could be the common pathogenesis $(5,6)$. Previous magnetic resonance imaging (MRI) studies discovered brain structural changes in patients with ESRD. Compared with the age-matched normal subjects, patients with ESRD had more white matter lesions (7), silent infarcts, cerebral atrophy (8), and axonal demyelination (9). Beyond the structural alterations, resting-state functional MRI (R-fMRI) recently has become a valuable tool in discovering functional changes of brain networks in patients with ESRD.

Resting-state functional MRI is based on the blood oxygen level-dependent (BOLD) principle at an intrinsic spontaneous (resting) status, where the R-fMRI signals can be analyzed to detect the temporal synchronizations across distant brain areas, referred to as functional connectivity (10). The alterations of functional connectivity in patients with ESRD may present with an extensive and variable pattern based on different analytical algorithms. Earlier, R-fMRI studies of patients with ESRD found decreased brain regional activity in a diffuse pattern over bilateral frontal, parietal, and temporal lobes, or impaired default-mode network (DMN) connectivity in the posterior cingulate cortex, precuneus, and medial prefrontal cortex $(11,12)$, without mention of dialysis modality. Subsequent studies have identified different aspects of connectivity reduction in patients with ESRD (13-16). Zheng et al. observed the decreased connectivity to be predominantly related to the prefrontal lobe in patients with ESRD receiving hemodialysis (HD), which involves planning complex cognitive behavior (13). Chen et al. found that patients with ESRD with HD had decreased regional homogeneity ( $\mathrm{ReHo}$ ) in DMN when compared to those without replacement therapy (14). Evidence on examining the global functional connectivity of ESRD patients with HD found abnormal intrinsic dysconnectivity pattern of salience network (SN) regions over bilateral insula and dorsal anterior cingulate cortex (15). However, most of the above ESRD studies were restricted to patients with HD with a certain extent of cognitive impairment.

The procedure of HD itself may cause significant systemic circulatory stress and cardiac stunning $(17,18)$. This repetitive stress may lead to persistent perfusion anomalies and accelerate end organ damage, including the brain (19, 20). Unlike $\mathrm{HD}$, the peritoneal dialysis (PD) procedure does not involve extracorporeal circulation, and patients with $\mathrm{PD}$ have a less hemodynamic impact based on their daily dialysis. Very few brain fMRI studies focused on patients with PD only and showed inconsistent results at the current stage. One previous study discovered that patients undergoing $\mathrm{PD}$ had lower values of the amplitude of low-frequency fluctuations (ALFF) in the parietal lobes and left precuneus (21). Recently, another study demonstrated that the patterns of functional connectivity among patients with PD and HD were not consistent. Compared to the healthy controls, only patients with HD but not patients with PD had significant changes in global functional connectivity. The authors concluded that the brain networks may be affected by different types of renal replacement therapy (22). Moreover, disruption of brain function may precede neurological symptoms. Hence, we aimed at assessing changes of connectivity in cognitively-intact patients with ESRD under regular PD therapy. We believed that recognizing connectivity change of neurologically asymptomatic patients with PD might help to detect subtle brain dysfunctions and identify susceptible brain regions in this specific group. Technically, we took two analytical strategies, global and local searches, to comprehensively present the altered integrity of brain functionality among patients with PD. Regarding the global search, the graph analysis was adopted to reveal the functional alterations of the wholebrain connectivity (23). In the local search, network-specific connectivity maps were analyzed to reveal the functional changes of multiple ESRD-related networks, such as DMN and salience network (SN) $(12,15)$. Furthermore, we also included the sensorimotor network (SMN) for the reported slow motor performances and the hippocampal network (HN) for the memory disturbances in the patients with ESRD on PD therapy $(4,21)$. 


\section{MATERIALS AND METHODS}

\section{Ethics Statement}

Written informed consent was obtained from all subjects. This study was conducted in accordance with the principles expressed in the Declaration of Helsinki and was approved by the Institutional Review Board of Chang Gung Memorial Hospital (IRB approval number: 102-1346A3).

\section{Patients and Clinical Evaluation}

This cross-sectional study recruited 19 patients with ESRD (including nine men) who received PD for at least 1 year and 24 age- and sex-matched controls (including nine men) with normal renal functions. All participants were right-handed. The causes of ESRD in the 19 patients included diabetic nephropathy in six patients, hypertensive nephropathy in five, chronic glomerulonephritis in four, renal artery stenosis in one, kidney graft failure in one, and unknown etiology in two patients. We excluded patients with known neurological disorders and psychiatric diseases, and those taking any medication involving the central nervous system (e.g., sleeping pills). Each subject underwent pain assessment with the numeric scales and depression screening based on Patient Health Questionnaire2 (PHQ-2) by a neurologist (T.Y.C.) at the outpatient clinic before the enrollment. Baseline mini-mental status examination (MMSE) score was evaluated by a licensed psychologist. Subjects with any evidence of pain, depressive mood, or with an MMSE score below 24 were also excluded $(24,25)$. A routine laboratory test for the patients was done within 1 week prior to performing the MRI scan. For the controls, basic biochemistry data was recorded upon enrollment. Each participant received compensation of $\sim$ US $\$ 20$.

\section{Image Acquisition}

Magnetic resonance imaging was conducted on a 3-T scanner (GE Healthcare, Milwaukee, WI) with T1-weighted images, T2weighted fluid-attenuated inversion recovery (FLAIR) image, and R-fMRI. The T1-weighted image was performed using the BRAVO sequence with $512 \times 512 \times 160$ matrix size; $0.5 \times 0.5 \times$ $1 \mathrm{~mm}$ resolution; inversion time $=450 \mathrm{~ms}$; repetition time $(\mathrm{TR})$ $=8,252 \mathrm{~ms}$; echo time $(\mathrm{TE})=3.2 \mathrm{~ms}$; flip angle $(\mathrm{FA})=12^{\circ}$; and $\mathrm{NEX}=1$. The T2-FLAIR sequence was performed to exclude anatomical lesions, especially the white matter hyperintensities, by using the following parameters: slice thickness $=3.5 \mathrm{~mm} ; 512$ $\times 512 \times 32$ matrix size; field of view $($ FOV $)=220 \times 220 \mathrm{~mm}$; TR $=9,000 \mathrm{~ms} ; \mathrm{TE}=140 \mathrm{~ms}$; inversion time $(\mathrm{TI})=2,250 \mathrm{~ms}$; and $\mathrm{FA}$ $=90^{\circ}$. R-fMRI was performed using a $\mathrm{T}_{2}^{*}$-weighted single-shot gradient-echo echo-planar imaging sequence $(\mathrm{TR} / \mathrm{TE} / \mathrm{FA}=2,000$ $\mathrm{ms} / 30 \mathrm{~ms} / 90^{\circ}, \mathrm{FOV}=220 \mathrm{~mm} \times 220 \mathrm{~mm}$; in-plane matrix $=64$ $\times 64$; in-plane resolution $=3.4 \times 3.4 \mathrm{~mm}$, and slice thickness $=4 \mathrm{~mm}$ ). For each patient, 36 continuous axial slices per volume and 180 volumes were acquired with an acquisition time of $6 \mathrm{~min}$. During R-fMRI scanning, the subjects were asked to keep their eyes closed, maintain head position, not fall asleep, and think about nothing in particular.

\section{Data Preprocessing}

Resting-state functional MRI data preprocessing was conducted using SPM8 (Welcome Department of Cognitive Neurology, Institute of Neurology, London, UK) and REST (26) in MATLAB (Mathworks, Natick, MA, USA) platform. All functional datasets underwent slice timing correction, realignment for head motion correction, spatial normalization to the Montreal Neurological Institute space (re-sampled to $2 \times 2 \times 2 \mathrm{~mm}^{3}$ of voxel size), and spatial smoothness with full-width half-maximum of $8 \mathrm{~mm}$. Head motion exceeding $\pm 3 \mathrm{~mm}$ of translation or $\pm 0.5 \mathrm{~mm}$ framewise displacement were excluded from the dataset. Subsequently, the linear trend was removed. Physiological noise from the CSF and white matter was regressed out, and also the motion parameters of maximum translation and framewise displacement were regressed out in each dataset [the maximum translation (mean \pm SD): Controls $=0.81 \pm 0.63 \mathrm{~mm}, \mathrm{PD}=1.05 \pm 0.58 \mathrm{~mm}$, nonsignificance; and the averaged framewise displacement (mean $\pm \mathrm{SD}$ ): Controls $=0.12 \pm 0.07 \mathrm{~mm}, \mathrm{PD}=0.16 \pm 0.08 \mathrm{~mm}$, non-significance]. Finally, the data were band-pass filtered at the frequency band of $0.01-0.08 \mathrm{~Hz}$.

\section{Global Connectivity: Graph Analysis}

Graph theory analysis constructs the brain regions ("nodes") and the connections between the regions ("edges") as a complex network, referred to as "connectivity," in a topological organization (27). To estimate the global change of brain networks, the pre-processed R-fMRI dataset was parcellated by the well-defined automated anatomical labeling (AAL) atlas into 90 regions (nodes) of interest for each patient (28) (see Supplementary Table 1). The mean time series of each region was then obtained by averaging the signal intensities over all voxels within a region of interest. After calculating the Pearson correlation coefficient $(r)$ between each pair of 90 regions of interest, a $90 \times 90$ correlation matrix was constructed for each individual. Cross-region correlation coefficients were regarded as unidirectional edges if $r$ values exceeded a threshold. Subsequently, specific $r$ thresholds were chosen to match fixed density values $(0.1,0.2$, and 0.3$)$, where the density was defined as connected edges divided by the maximum number of possibly connected edges over the entire brain. The density range of 0.1-0.3 was found to be reliable in the R-fMRI literature (29). Thus, a binary connectivity matrix was generated after the $r$ thresholding, and the graph analysis was applied to the binary matrix to estimate the whole-brain network properties using brain connectivity toolbox (30). The node-based network properties included degree, clustering coefficient (C), local efficiency $\left(\mathrm{E}_{\mathrm{loc}}\right)$, and betweenness centrality $(\mathrm{BC})$, and the global network properties included characteristic path length (L), global efficiency $\left(E_{\text {glob }}\right)$, modularity $(Q)$, small-worldness $(\sigma)$, and assortativity coefficient (A). Supplementary Figure 1 shows the topological parameters of graph theory used in this study. Mathematical definitions of these parameters are according to the study by Rubinov and Sporns (30), and the brief descriptions and illustrations of the meanings of these parameters are provided in Supplementary Material. 


\section{Network-Specific Connectivity: Seed-Based Analysis}

As the large AAL regions could dilute the temporal coherence and minimize the spatial specificity, we further adopted the seedbased analysis to measure the functional alterations of the four brain networks. Spherical seeds with a radius of $4 \mathrm{~mm}$ were prescribed from four target networks for functional evaluations: the DMN [seed at posterior cingulate cortex: $(0,-51,30)$ ], the SMN [seed at right precentral gyrus: $(36,-25,57)$ ], the SN [seed at right insula: $(30,9,10)]$, and the $\mathrm{HN}$ [seed at right anterior hippocampi: $(24,-20,-22)]$ (31). Time courses averaged within the seeds were used as a reference to perform correlation analysis over the entire brain. The resulting correlation coefficients were converted using Fisher's $z$-transform, resulting in the connectivity map for the subsequent group analysis.

\section{Intrinsic Brain Activity: Amplitude of Low-Frequency Fluctuations}

Beyond the connectivity changes, we also analyzed additional R-fMRI index of intrinsic brain activity, ALFF, in both patient and control groups. The related details are provided in Supplementary Material.

\section{Statistical Analysis}

Demographic and clinical data between the two groups were compared using the chi-squared test or independent $t$-test. An independent $t$-test was used for comparing the graph analysis parameters and connectivity networks between the two groups. Linear regression and Pearson correlation coefficient $r$ were applied for evaluating the relationship between clinical data and graph parameters. These statistical analyses were performed using the Statistical Package for Social Sciences [IBM SPSS Statistics for Mac, version 24], and the results were considered significant at $p<0.05$. For the group comparison of connectivity maps, we used AFNI software (32). Considering multiple corrections in fMRI results, the one-sample group average was displayed with family-wise error (FWE)-corrected $p<0.05$ with cluster threshold of 20 voxels, whereas the between-group contrast maps were demonstrated with $3 \mathrm{dClustSim-corrected} p$ $<0.05$. The multiple comparisons in the group analysis were corrected through the $3 \mathrm{dClustSim}$ approach with the autocorrelation function, and the significance level was $p<0.05$ (uncorrected $p<0.001$, cluster threshold $=103$ voxels).

\section{RESULTS}

\section{Demographic Data}

Table 1 shows the demographic characteristics of the two groups. The education years of patients and the age- and sex-matched controls were $13.4 \pm 3.3$ years vs. $11.1 \pm 4.3$ years $(p=$ 0.06), respectively, and the two groups had similar MMSE performance ( 28 vs. 28.9, $p=0.13$ ), which represented no clinically overt cognitive impairment. Among the patient group, the average duration of receiving PD was $4.2 \pm 3.7$ years. The PD duration for each patient is listed in Supplementary Table 2. As hypertension and diabetes mellitus (DM) could influence intrinsic brain activities $(33,34)$, to account for this issue, we enrolled controls and patients with ESRD with similar
TABLE 1 | Demographic characteristics of subjects.

\begin{tabular}{|c|c|c|c|}
\hline Characteristics & $\begin{array}{c}\text { PD } \\
(n=19)\end{array}$ & $\begin{array}{l}\text { Controls } \\
(n=24)\end{array}$ & $P$ \\
\hline Sex, male (\%) & 47.4 & 37.5 & $0.5^{\mathrm{a}}$ \\
\hline Age (mean $\pm S D$ ) & $49.8 \pm 13$ & $51.2 \pm 7.6$ & $0.6^{\mathrm{a}}$ \\
\hline Education, years (mean \pm SD) & $13.4 \pm 3.3$ & $11.1 \pm 4.3$ & $0.06^{\mathrm{a}}$ \\
\hline Duration of PD, years (mean $\pm S D)^{\star}$ & $4.2 \pm 3.7$ & $N / A$ & $\mathrm{~N} / \mathrm{A}$ \\
\hline MMSE score & $28.2 \pm 1.5$ & $28.9 \pm 1.6$ & $0.1^{\mathrm{a}}$ \\
\hline Cause of ESRD & Variable ${ }^{\star \star}$ & $\mathrm{N} / \mathrm{A}$ & $\mathrm{N} / \mathrm{A}$ \\
\hline \multicolumn{4}{|c|}{ Chronic illness and personal history, presence $[n,(\%)]$} \\
\hline Hypertension & $16 / 19(84)$ & $14 / 24(58)$ & $0.07^{b}$ \\
\hline Hyperlipidemia & $4 / 19(21)$ & $4 / 24(17)$ & $0.99^{b}$ \\
\hline CAD & $2 / 19(11)$ & 2/24 (8) & $0.81^{b}$ \\
\hline $\mathrm{DM}$ and pre- $\mathrm{DM}^{+}$ & $8 / 19(42)$ & 8/24 (33) & $0.56^{b}$ \\
\hline Alcohol ++ & 0/19 (0) & 0/24 (0) & $1.0^{\mathrm{b}}$ \\
\hline \multicolumn{4}{|l|}{ Laboratory data (mean \pm SD) } \\
\hline White blood cell count, $10^{12} / \mathrm{L}$ & $7.5 \pm 1.7$ & $\mathrm{~N} / \mathrm{A}$ & $\mathrm{N} / \mathrm{A}$ \\
\hline Hemoglobin, g/dl & $10.7 \pm 0.9$ & $\mathrm{~N} / \mathrm{A}$ & $\mathrm{N} / \mathrm{A}$ \\
\hline Hematocrit, \% & $31.7 \pm 2.7$ & $\mathrm{~N} / \mathrm{A}$ & $\mathrm{N} / \mathrm{A}$ \\
\hline Albumin, g/dl & $3.8 \pm 0.4$ & $\mathrm{~N} / \mathrm{A}$ & $\mathrm{N} / \mathrm{A}$ \\
\hline Uric acid, mg/dl & $7.0 \pm 0.7$ & $6.1 \pm 1.6$ & $0.02^{\mathrm{a}, \#}$ \\
\hline $\mathrm{HbA} 1 \mathrm{C}, \%$ & $5.9 \pm 0.8$ & $5.5 \pm 0.5$ & $0.13^{\mathrm{a}}$ \\
\hline Total cholesterol, mg/dl & $175 \pm 27.8$ & $186 \pm 36.3$ & 0.29 \\
\hline LDL-cholesterol, (mg/dl) & $119 \pm 26.7$ & $105 \pm 32.2$ & 0.22 \\
\hline HDL-cholesterol (mg/dl) & $45 \pm 13.9$ & $51 \pm 14.7$ & 0.16 \\
\hline Triglyceride (mg/dl) & $141 \pm 59.9$ & $144 \pm 99.9$ & 0.89 \\
\hline
\end{tabular}

The demographic comparisons between the two groups: ${ }^{a}$ independent $t$-test; ${ }^{b}$ chisquare test.

Significance is defined when $p<0.05$. ${ }^{*} p<0.05$.

*The duration of undergoing dialysis for each patient is listed in Supplementary Material.

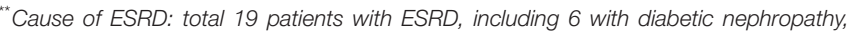
5 with hypertensive nephropathy, 4 with chronic glomerulonephritis, 1 with renal artery stenosis, 1 with graft failure, and 2 with unknown etiology.

+ pre-DM: defined as $\mathrm{HbA} 1 \mathrm{C}>5.7 \%$.

++ Alcohol: alcohol use more than "drinking in moderation" (defined as <2 drinks in a day by the "Dietary Guidelines for Americans 2020-2025," U.S. Department of Health and Human Services and U.S. Department of Agriculture).

$P D$, peritoneal dialysis; MMSE, mini-mental status examination; ESRD, end-stage renal disease; CAD, coronary artery disease; DM, diabetes mellitus; N/A, not applicable.

proportions of chronic diseases. The subjects from both groups had comparable baseline characteristics among the percentage of major systemic diseases (hypertension, coronary artery disease, and $\mathrm{DM}$ ), glycated hemoglobin (HbA1C), and lipid profiles. Compared to the controls, the patients had a higher level of uric acid (7.0 \pm 0.7 vs. $6.1 \pm 1.6, p=0.02)$. Data of white blood cell count, hemoglobin, hematocrit, and albumin were only available for the patient group.

\section{Graph Analysis}

The AAL-based edges are shown in Figure 1A (density $=0.2$ ) for both groups. Overall, the connections (edges) were denser in the control group when compared to the PD group. Table 2 and Figure 1B demonstrate the nine network properties from graph analysis. Compared with the controls, the patients with ESRD had stronger BC (PD: $110.54 \pm 16.71$ vs. controls: $97.39 \pm 14.45, p<0.01$ ) and L (PD: $2.31 \pm 0.23$ vs. controls: $2.12 \pm 0.13, p<0.01)$. Meanwhile, the controls had 


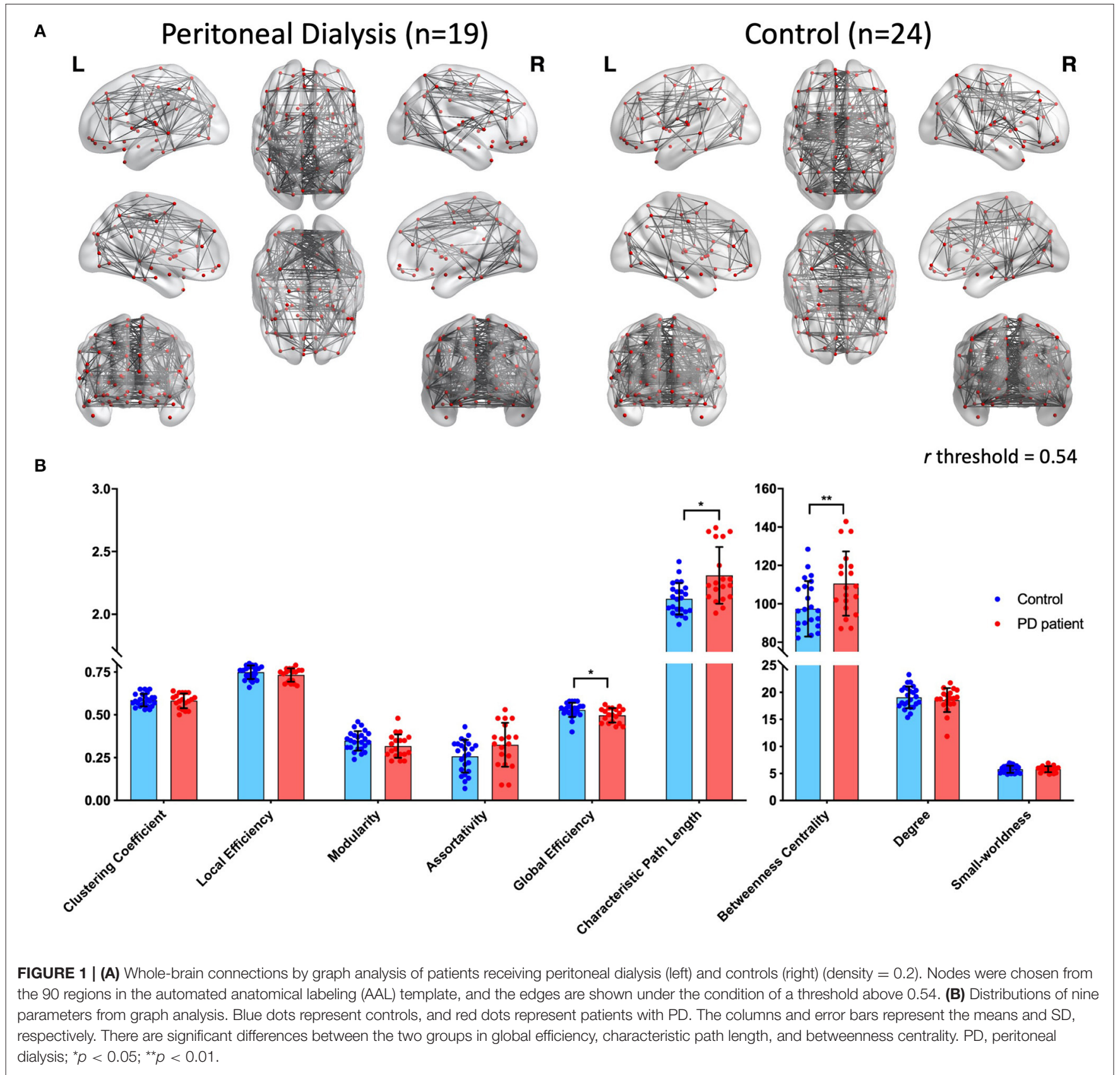

higher $\mathrm{E}_{\text {glob }}$ than the patients (PD: $0.50 \pm 0.04$ vs. controls: $0.53 \pm 0.04, p<0.05)$.

\section{Seed-Based Connectivity}

Four networks were chosen for functional alterations in patients with ESRD through the seed-based connectivity analysis, including DMN (Figure 2), SMN (Figure 3), SN (Figure 4), and HN (Figure 5). The brain areas with significant differences indicated that their functional connection with the seed region changed in the patient group when compared with the controls. The regional details of the connectivity changes are described below and summarized in Table 3.

\section{DMN}

Figure 2 shows the DMN connectivity maps of the patients and controls. Compared with the controls, the patients with ESRD had lesser functional connectivity, mainly at the temporal gyri, anterior cingulate cortex, superior frontal gyrus, and precuneus; meanwhile, they also showed increased connectivity at the insula, middle frontal gyrus, and inferior parietal lobe.

\section{SMN}

In SMN (Figure 3), the patients had decreased functional connectivity at the insula and parahippocampal gyrus. 
TABLE 2 | Comparison of parameters from graph analysis.

\begin{tabular}{lccc}
\hline $\begin{array}{l}\text { Density }=\mathbf{0 . 2} \\
\text { Parameters }\end{array}$ & $\begin{array}{c}\text { PD } \\
(\boldsymbol{n}=\mathbf{1 9 )} \\
\text { (mean } \pm \text { SD) }\end{array}$ & $\begin{array}{c}\text { Control } \\
(\boldsymbol{n}=\mathbf{2 4}) \\
(\mathbf{m e a n} \pm \mathbf{S D})\end{array}$ & $\boldsymbol{p}^{\text {-value }}$ \\
\hline Degree & $18.58 \pm 2.22$ & $19.09 \pm 2.01$ & 0.443 \\
Clustering coefficient & $0.58 \pm 0.04$ & $0.59 \pm 0.04$ & 0.766 \\
Local efficiency & $0.73 \pm 0.04$ & $0.75 \pm 0.04$ & 0.191 \\
Betweenness centrality & $110.54 \pm 16.71$ & $97.39 \pm 14.45$ & $0.008^{\star \star}$ \\
Characteristic path length & $2.31 \pm 0.23$ & $2.12 \pm 0.13$ & $0.001^{\star \star}$ \\
Global efficiency & $0.50 \pm 0.04$ & $0.53 \pm 0.04$ & $0.019^{\star}$ \\
Modularity & $0.32 \pm 0.07$ & $0.35 \pm 0.06$ & 0.116 \\
Small-worldness & $5.80 \pm 0.55$ & $5.79 \pm 0.66$ & 0.945 \\
Assortativity & $0.33 \pm 0.13$ & $0.26 \pm 0.10$ & 0.054
\end{tabular}

+ Independent $t$-test was used for between-group comparisons. Significance is defined when $p<0.05$.

${ }^{*} p<0.05$.

${ }^{\star \star} p<0.01$.

\section{SN}

In SN (Figure 4), the patients had significantly increased functional connectivity at the thalamus and decreased connectivity at the superior temporal gyrus, anterior cingulate gyrus, and postcentral gyrus when compared to controls.

\section{$\mathrm{HN}$}

The HN of the patients and controls is shown in Figure 5. The controls had stronger functional connectivity than the patients in the precentral gyrus, postcentral gyrus, superior temporal gyrus, and parahippocampal gyrus.

\section{Correlation Between Uric Acid and the Global Connectivity}

Since the uric acid levels showed significant differences between the patients and the controls, correlation analysis was performed to evaluate the relationship between uric acid and selected global connectivity metrics in the patient group and in all subjects. Linear regression by Pearson correlation coefficient showed no significant correlation between uric acid and global connectivity of $\mathrm{BC}, \mathrm{L}$, and $\mathrm{E}_{\mathrm{glob}}$, either in the patient group or in all subjects. The correlation data is summarized in Supplementary Table 3.

\section{The Amplitude of Low-Frequency Fluctuations}

The comparison of ALFF between both groups revealed no significant differences. The corresponding result is shown in Supplementary Figure 2.

\section{DISCUSSION}

We examined the brain connectivity of cognitively intact patients with ESRD undergoing PD and compared it with age- and sexmatched controls. Our findings suggested that patients with PD had functional connectivity profiles that were significantly different from controls, based on both graph analysis and seed-based network analysis. To the best of our knowledge, this is the first brain connectivity report including both graph theory analysis and seed-based networks data in patients with PD. Unlike HD, in which intradialytic hypotension is very common, patients with PD have less hemodynamic stress during their routine dialysis therapy (35). The disruption of functional connectivity in our patients might not be mainly caused by hemodynamic alterations.

In the review of literature, there were two R-fMRI studies focusing on patients with ESRD undergoing PD. The first study enrolled patients with impaired cognitive performance and the R-fMRI analysis used ALFF as the only indicator of network changes (21). Compared to the healthy controls, patients with PD showed lower ALFF values in the bilateral frontal and parietal lobes, especially in the left precuneus, left inferior parietal lobe, and left superior parietal lobe. The other study performed graph theoretical analysis on both structural and functional connectivity. In global connectivity, patients with PD only showed decreased "structural" connectivity but not "functional" connectivity, while in local connectivity, patients with PD had variable changes (including increased and decreased connectivity) in $\mathrm{BC}$ over right frontal operculum, right inferior temporal, right temporal cortex pole, right occipital pole, right supracalcarine, left insular cortex, and left postcentral region (22). It was noted that most patients with PD in that study also had cognitive impairment, especially in frontal/executive function. In our study, all subjects maintained good daily functions without clinical cognitive decline, and the comprehensive analysis of spontaneous brain activities helped to reveal subtle changes in the brain circuits in patients with PD. Instead of referring to specific regions, our graph analysis of the functional connections was examined in a general view, and the results showed increased BC, increased L, and decreased $\mathrm{E}_{\text {glob }}$ in the PD group. BC denotes the influence of a "node" (a brain region) on the flow of information between all other nodes (36). Stronger BC implies the enrichment of hub-like nodes in the brain of patients (i.e., multiple nodes control the information flow, so the connecting structure tends to be dispersed). The path length, $\mathrm{L}$, is the average of all the shortest paths between all the node pairs in the brain, whereas the inverse measurement of $\mathrm{L}$ is $\mathrm{E}_{\text {glob }}(30)$. The high $\mathrm{L}$ value in the patients with $\mathrm{PD}$ insinuates relative estrangement between the nodes within the network, leading to potential inefficiency in regional communication. This was also evident in low $\mathrm{E}_{\text {glob }}$ in the patients, indicating the impaired ability of a network to communicate globally. Briefly, functional connectivity on graph analysis in our ESRD PD patients tended to show increased hub-nodes (higher $\mathrm{BC}$ ), more distant connections between the nodes (longer L), and less efficient global communication (lower $\mathrm{E}_{\text {glob }}$ ). We suggested that the above graph metrics could be indicators for identifying global connectivity alterations in patients with PD.

The seed-based analysis for functional connectivity investigates the temporal synchronizations between brain regions (37), and the four networks of DMN (encompassing medial prefrontal cortex, posterior cingulate cortex, bilateral inferior parietal cortex, and bilateral superior temporal lobes) (31, 38), SMN (encompassing bilateral primary motor cortex and supplementary motor area) (39), SN (encompassing dorsal anterior cingulate cortex and bilateral anterior insula) (15), and 

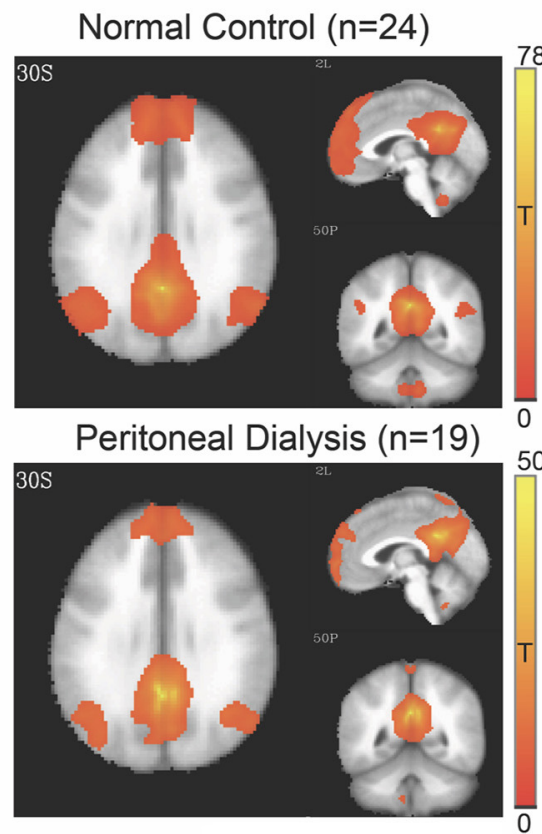

Contrast (NC>PD)

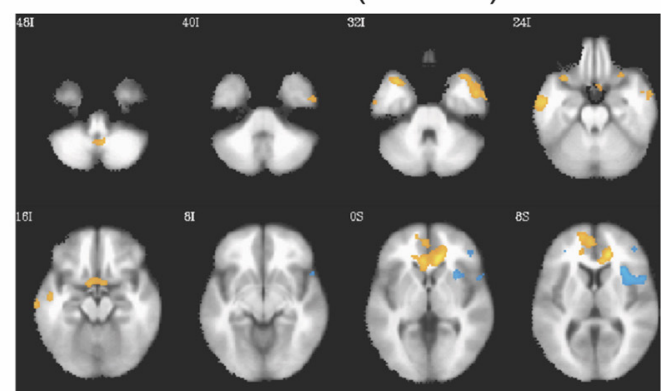

408

325
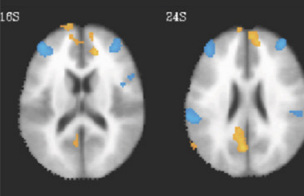

$56 \mathrm{~S}$
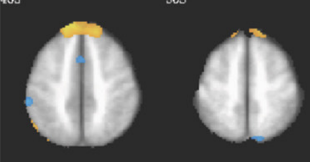

(1)

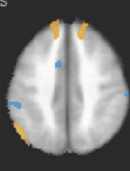

728

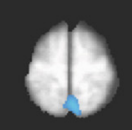

FIGURE 2 | Default-mode network (DMN) of controls and patients with PD. Connectivity map of either group is shown at left side (FWE-corrected $p<0.05)$, and the contrast at right side (3dClustSim-corrected $p<0.05)$. In the contrast map, the orange color represents significantly increased connectivity in controls, including anterior cingulate gyrus, superior and medial frontal gyrus, temporal lobe, and precuneus, whereas the blue color represents decreased connectivity in controls, including the insula, inferior parietal lobule, middle frontal gyrus, and supplemental motor area. PD, peritoneal dialysis; NC, normal controls.
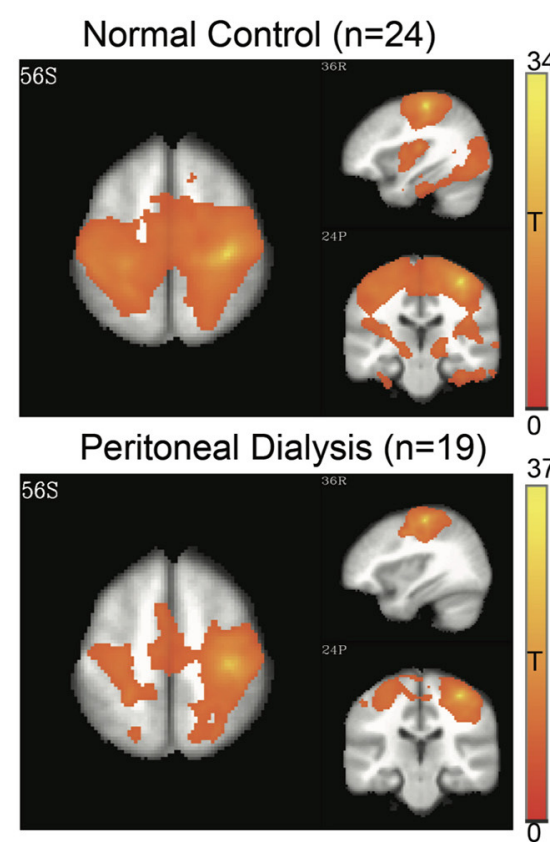

Contrast $(\mathrm{NC}>\mathrm{PD})$

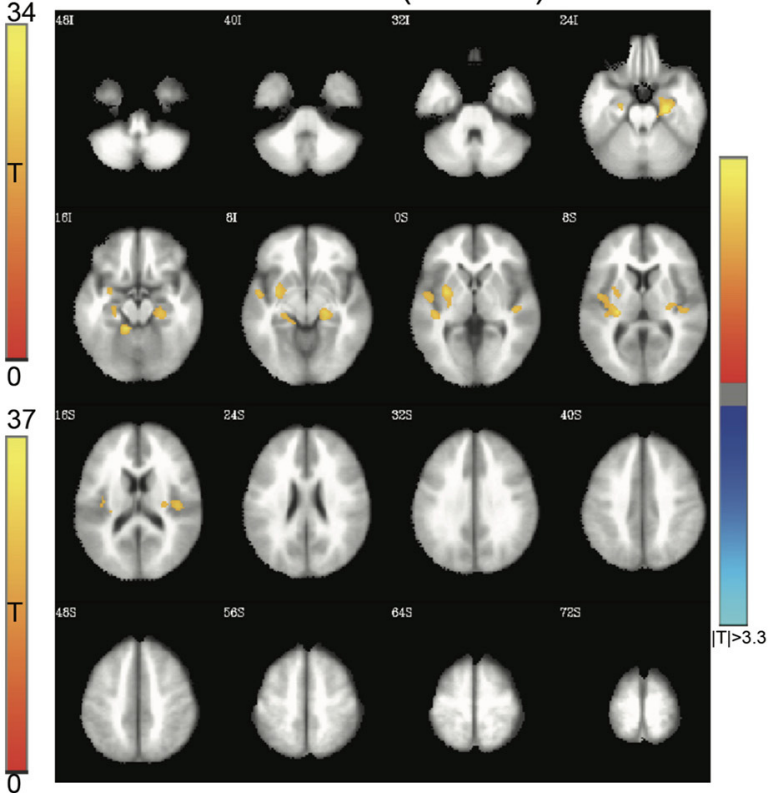

FIGURE 3 | Sensorimotor network (SMN) of controls and patients with PD. Connectivity map of either group is shown at the left side (FWE-corrected $p<0.05$ ), and the contrast map at the right side (3dClustSim-corrected $p<0.05)$. In the contrast map, the orange color represents significantly increased connectivity in controls, including parahippocampal gyrus and insula. PD, peritoneal dialysis; NC, normal controls. 

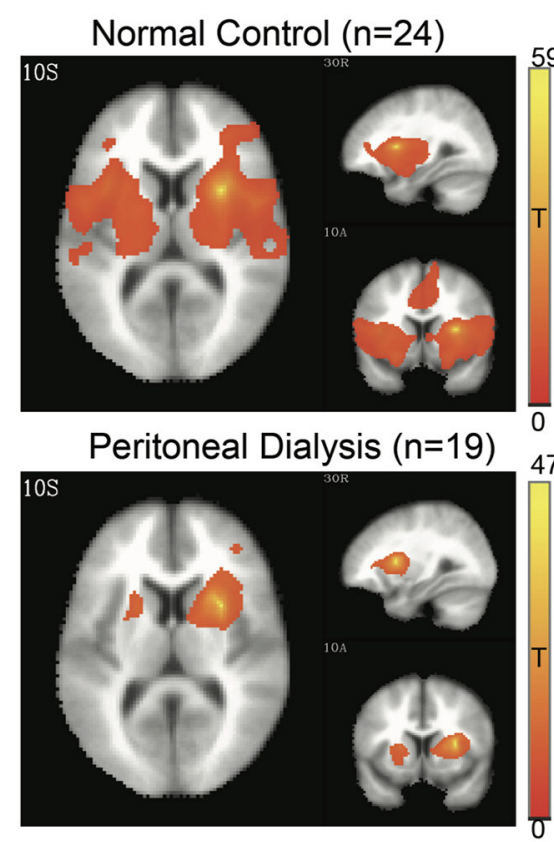

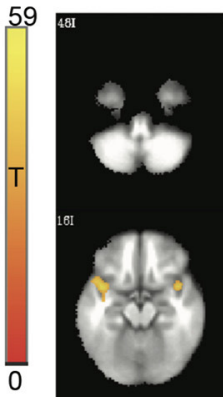

as
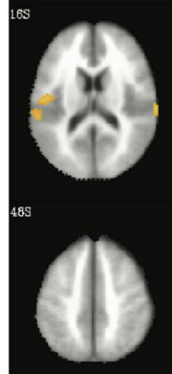

Contrast (NC>PD)

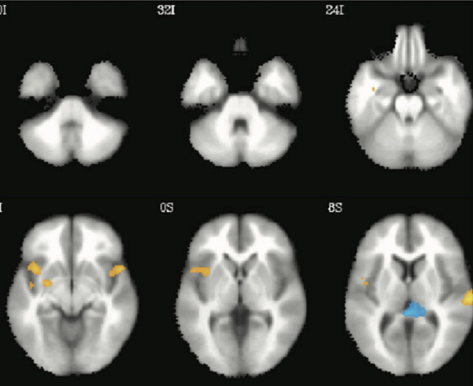

245
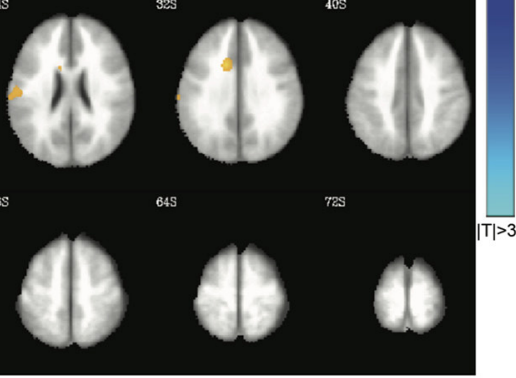

FIGURE 4 | Salience network (SN) of controls and patients with PD. Connectivity map of either group is shown at the left side (FWE-corrected $p<0.05$ ), and the contrast map at the right side (3dClustSim-corrected $p<0.05)$. In the contrast map, the orange color represents significantly increased connectivity in controls, including temporal gyrus, cingulate gyrus, and postcentral gyrus, whereas the blue color represents decreased connectivity in controls, mainly in the thalamus. PD, peritoneal dialysis; NC, normal controls.
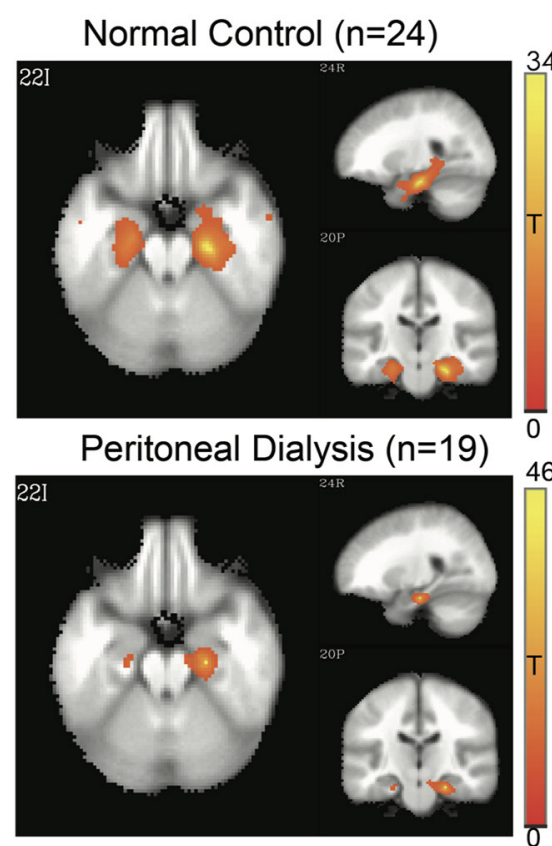

\section{Contrast (NC>PD)}
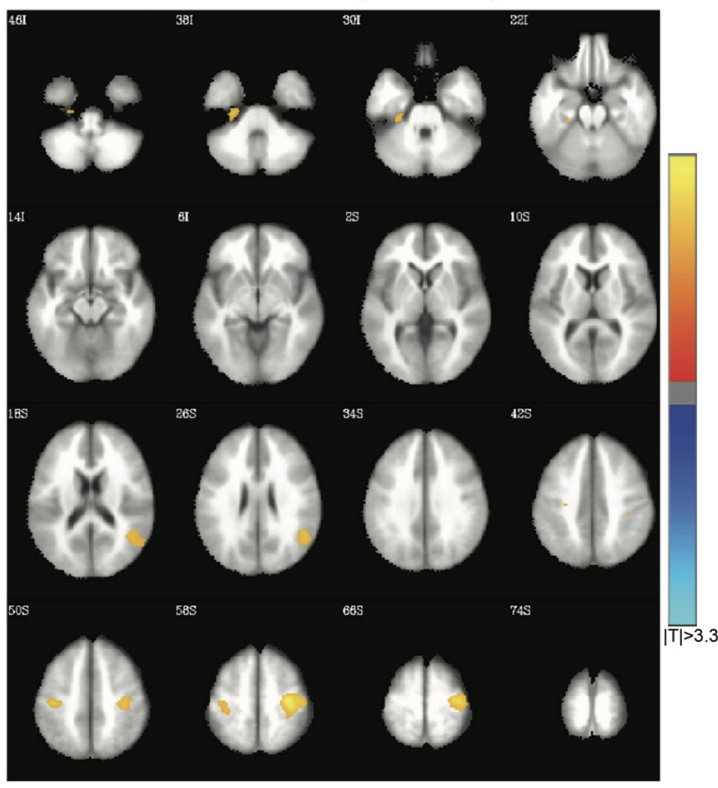

FIGURE 5 | Hippocampal network (HN) of controls and patients with PD. Connectivity map of either group is shown at the left side (FWE-corrected $p<0.05)$, and contrast map at the right side (3dClustSim-corrected $p<0.05)$. In the contrast map, the orange color represents significantly increased connectivity in controls, whereas the blue color represents decreased connectivity in controls. PD, peritoneal dialysis; NC, normal controls. 
TABLE 3 | Brain regions with significant connectivity differences between PD and controls of selected functional networks.

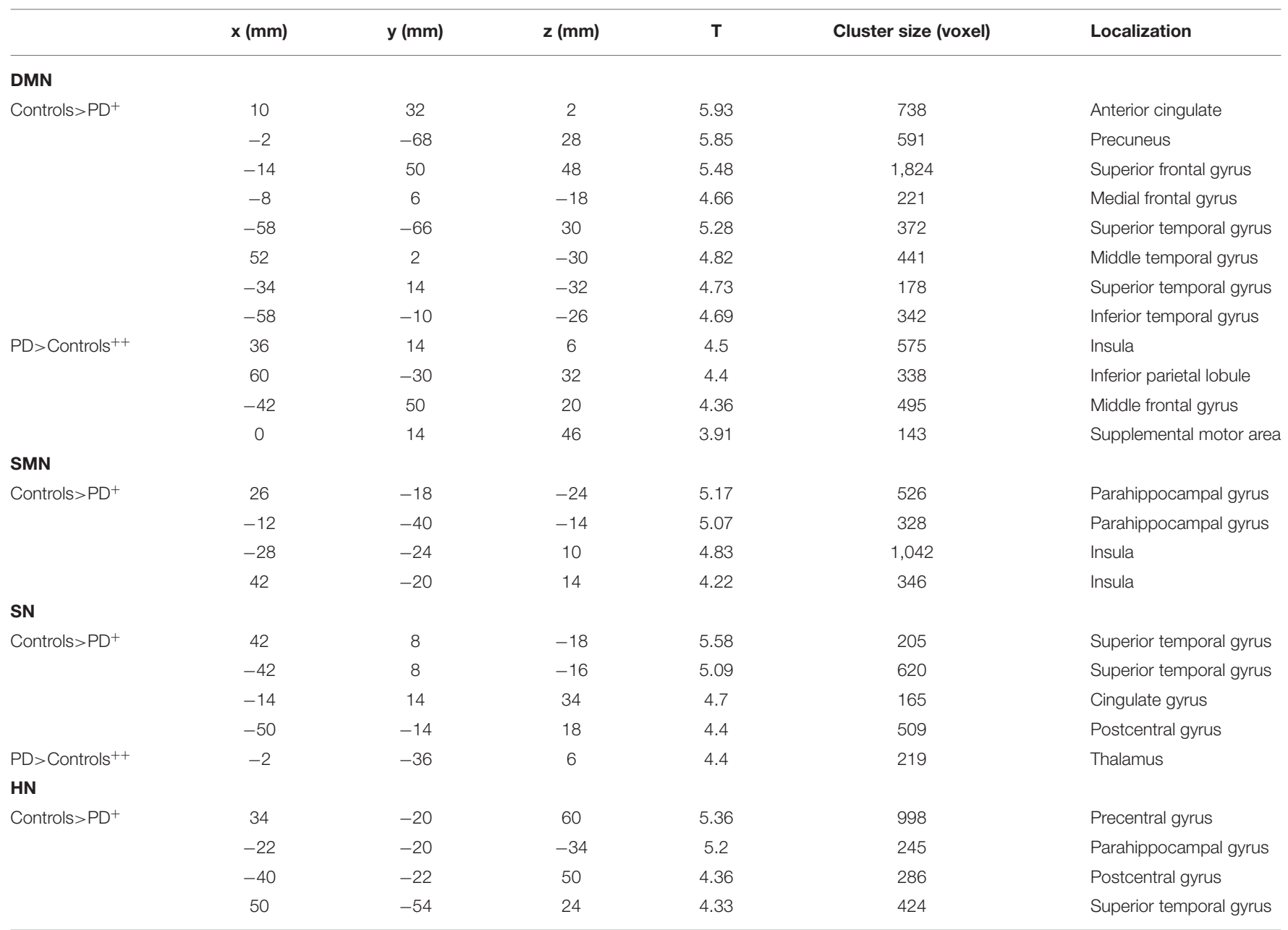

PD, peritoneal dialysis; T, peak t-value; DMN, default-mode network; SMN, sensorimotor network; SN, salience network; HN, hippocampal network (p < 0.05, 3dClusterSim-corrected). + Controls > PD: significantly stronger connectivity of normal controls.

$++P D>$ Controls: significantly stronger connectivity of patients with peritoneal dialysis.

HN (encompassing bilateral hippocampi) (40) are commonly evaluated for main brain activities. In DMN, the patients with PD in our study had significantly decreased connectivity at the anterior cingulate cortex, superior and medial frontal gyrus, temporal lobe, and precuneus. A previous study found decreased DMN connectivity at similar areas in patients with ESRD (12), without specifying the type of dialysis. DMN is believed to be involved in self-projection and self-awareness (41). The medial prefrontal part is related to episodic memory (42), and the precuneus may be responsible for supporting autobiographical memory and integrating complex information (43). Our findings, which were in agreement with previous studies, demonstrated reduced DMN connectivity, mainly at the medial frontal and precuneus regions. The data indicate that reduced intrinsic brain connections in patients with ESRD can be observed under the condition without clinical cognitive impairment. Furthermore, our patients also had decreased DMN connectivity at the anterior cingulate gyrus, the middle and inferior temporal lobes, which are partially consistent with two earlier reports in patients with ESRD, without mentioning cognitive decline $(14,44)$. The anterior cingulate cortex is believed to be involved in affect regulation and the ability to manage unpleasant emotions (45). Whether decreased connectivity at these areas is linked to clinical significance requires further longitudinal follow-up.

Interestingly, our DMN data also revealed increased connectivity at the inferior parietal lobule, middle frontal gyrus, supplemental motor area, and insula in the patients, where the supplemental motor area and insula belong to other functional networks such as SMN and SN, respectively. Such cross-network connectivity changes could indicate implicit neurophysiological alterations for sensorimotor or pain perception. For the pain perception, Coppola et al. demonstrated that greater subjective intensity of pain during a migraine attack was associated with weaker DMN-insula connectivity (46), and Usui et al. also presented that the DMN-insula connectivity changes correlated with the difference in pain scores of patients with fibromyalgia after music intervention (47). Chronic pain is common among the ESRD population but is usually underestimated (48). In our 
hospital, pain assessment was mandatory during an outpatient clinic visit. Although none of the participants in our study reported pain upon enrollment, chronic sensory discomfort was not further evaluated and pain-related activation could not be completely excluded. On the other hand, while the insula and middle frontal gyrus had increased connectivity in the patients with PD in our study, another study showed decreased connectivity in these brain regions in ESRD HD patients (14). The underlying mechanisms of this discrepancy is not fully understood and different renal replacement therapy could play a role. Furthermore, in contrast to the decreased within-DMN connectivity in the patients with ESRD (49), our results denoted the increased DMN-insula connectivity. Based on our experiences, such specific connectivity changes of DMN or SN along with neuropathology could be associated with the alterations (either deteriorative or compensatory) of cognitive performances $(39,50)$. However, future studies are warranted to verify such inference due to the lack of cognitive tasks in this study.

The current study also found some alterations of $\mathrm{SN}$ connectivity in the patient group. $\mathrm{SN}$ involves the responses to behaviorally salient events. The patients in our study displayed lower between-network connectivity at the cingulate and postcentral gyri in $\mathrm{SN}$, while insula-thalamus connectivity was clearly increased in the patients with ESRD receiving PD (Table 3), which has never been reported before. In contrast, a recent study that focused on patients receiving HD found hypoconnectivity in the thalamo-cortical network (16). Based on the observations, the SN could be a potential network susceptible to the changes of between-network connectivity in the patients with ESRD receiving PD; however, further investigation is needed to delineate whether dialysis modalities affect the patterns of connectivity alterations.

The patients in our study showed decreased SMN connectivity in the parahippocampal gyrus and insula. In a previous study, decreased SMN has been reported in the precentral and postcentral gyrus (51). SMN is a network associated with sensory input or motor coordination, which is activated by corresponding movement-demanding tasks (52). It is also possibly involved in movement coordination (53). Interestingly, the hippocampus in the patients with ESRD showed a reduced connection to the bilateral SMN, which was rarely observed in this field. Previous papers demonstrated the altered hippocampusthalamus connection and sensorimotor connectivity separately among neurological and psychiatric patients $(54,55)$. The reduced hippocampus-SMN connection in the ESRD might be associated with the memory disturbances and slow motor performances in the patients with ESRD, which requires further studies along with memory and movement performances in the future.

In relation to the demographic profiles of our subjects, patients with ESRD had significantly higher uric acid levels when compared to the controls. There is no published data about altered functional connectivity in hyperuricemia subjects. Only one study mentioned the positive correlation between the uric acid level and substantia nigra connectivity (56). In our cohort, no correlation was found between uric acid level and graph metrics (Supplementary Table 3). Uric acid may not play an important role in connectivity change among the patients in our study.

Beyond the connectivity alterations, we performed extra analysis for intrinsic brain activity, the ALFF, which showed no difference between the groups in the current study. Previously, Luo's study (21) showed that patients with ESRD receiving PD had a reduction in ALFF at the inferior parietal lobe. This discrepancy could be related to the clinical profiles. Although the patients in our study did not receive detailed neuropsychological assessment, they did exhibit potent daily functions and good MMSE performance. Patients in Lou's study had cognitive impairments, and their controls did not have any systemic illness. The controls in our study had corresponding diseases as patients with ESRD since underlying chronic illness may influence intrinsic brain activities $(33,34)$. ALFF may be sensitive to biological changes in the brain activity of patients with ESRD, but this was not evident in our patient group, perhaps because of fair cognitive function and comparative controls.

\section{LIMITATIONS}

The main shortcoming of our study was the relatively small sample size. Second, the neuropsychological assessment was incomprehensive, and there was a marginally statistical significance of education years between the two groups. It was difficult to delineate the affected brain regions on connectivity to a respective neuropsychological function domain in the patients in our study. Recruitment of more subjects with a further comparison of functional connectivity between patients with ESRD with different replacement therapies as well as a detailed cognitive assessment should be conducted. Third, a detailed clinical evaluation of pain or paresthesia was not performed. Sensory discomfort should be carefully reviewed in the following ESRD R-fMRI studies. At last, anemia is very frequently observed in patients with ESRD. Literature has shown the influence of hemoglobin on connectivity (57). However, we did not perform the hemoglobin comparison since hemoglobin was not routinely checked for the controls. The possible effect of hemoglobin on connectivity could not be completely excluded.

\section{CONCLUSION}

We intensively demonstrated the various changes of the brain connectivity in patients with ESRD who underwent PD and preserved favorable cognitive function. In graph analysis, $\mathrm{E}_{\mathrm{glob}}$, $\mathrm{BC}$, and $\mathrm{L}$ were found to be potentially useful metrics to identify the connectivity changes since patients with ESRD showed significantly lower $\mathrm{E}_{\text {glob }}$ and higher BC and L. Networks including DMN, SMN, SN, and $\mathrm{HN}$ all showed alterations in the patients with ESRD, while elevated connectivity strength was observed only in DMN and SN. In patients with ESRD receiving $\mathrm{PD}, \mathrm{DMN}$ and SN might show more prominent connectivity alterations. Changes in cerebral connectivity may develop without a clinical decline of cognition. Further longitudinal 
follow-up is required to clarify whether asymptomatic patients with connectivity disruption are prone to have cognitive impairment or dementia later.

\section{DATA AVAILABILITY STATEMENT}

The original contributions presented in the study are included in the article/Supplementary Material, further inquiries can be directed to the corresponding author/s.

\section{ETHICS STATEMENT}

The studies involving human participants were reviewed and approved by Institutional Review Board of Chang Gung Memorial Hospital (IRB Approval Number: 102-1346A3). The patients/participants provided their written informed consent to participate in this study.

\section{AUTHOR CONTRIBUTIONS}

T-YC, H-HW, and CW: manuscript writing. T-YC, C-HY, and H-SJ: data acquisition. H-LL, H-SJ, and CW: data analysis. T-YC, H-HW, Y-JL, K-LH, T-HL, and Y-CT: data interpretation and literature review. H-LL, T-HL, Y-CT, and CW: research

\section{REFERENCES}

1. Wu HH, Kuo CF, Li IJ, Weng $\mathrm{CH}$, Lee $\mathrm{CC}, \mathrm{Tu} \mathrm{KH}$, et al. Family aggregation and heritability of ESRD in Taiwan: a population-based study. Am J Kidney Dis. (2017) 70:619-26. doi: 10.1053/j.ajkd.2017. 05.007

2. Nickolas TL, Khatri M, Boden-Albala B, Kiryluk K, Luo X, GervasiFranklin $\mathrm{P}$, et al. The association between kidney disease and cardiovascular risk in a multiethnic cohort: findings from the Northern Manhattan Study (NOMAS). Stroke. (2008) 39:2876-9. doi: 10.1161/STROKEAHA.107.5 13713

3. Post JB, Jegede AB, Morin K, Spungen AM, Langhoff E, Sano M. Cognitive profile of chronic kidney disease and hemodialysis patients without dementia. Nephron Clin Pract. (2010) 116:c247-55. doi: 10.1159/0003 17206

4. Kalirao P, Pederson S, Foley RN, Kolste A, Tupper D, Zaun D, et al. Cognitive impairment in peritoneal dialysis patients. Am J Kidney Dis. (2011) 57:61220. doi: 10.1053/j.ajkd.2010.11.026

5. Mogi M, Horiuchi M. Clinical interaction between brain and kidney in small vessel disease. Cardiol Res Pract. (2011) 2011:306189. doi: 10.4061/2011/306189

6. Bugnicourt JM, Godefroy O, Chillon JM, Choukroun G, Massy ZA. Cognitive disorders and dementia in CKD: the neglected kidney-brain axis. J Am Soc Nephrol. (2013) 24:353-63. doi: 10.1681/ASN.20120 50536

7. Khatri M, Wright CB, Nickolas TL, Yoshita M, Paik MC, Kranwinkel G, et al. Chronic kidney disease is associated with white matter hyperintensity volume: the Northern Manhattan Study (NOMAS). Stroke. (2007) 38:31216. doi: 10.1161/STROKEAHA.107.493593

8. Drew DA, Bhadelia R, Tighiouart H, Novak V, Scott TM, Lou KV, et al. Anatomic brain disease in hemodialysis patients: a cross-sectional study. Am J Kidney Dis. (2013) 61:271-8. doi: 10.1053/j.ajkd.2012. 08.035

9. Chou MC, Hsieh TJ, Lin YL, Hsieh YT, Li WZ, Chang JM, et al. Widespread white matter alterations in patients with end-stage renal disease: a mentorship and supervision. All authors contributed important intellectual content during manuscript drafting or revision and accepts accountability for the overall work by ensuring that questions pertaining to the accuracy or integrity of any portion of the work are appropriately investigated and resolved.

\section{FUNDING}

This work was supported by two grants (CMRPG3G0271 and CMRPG3A1041) from the Chang Gung Medical Research Council. The funders had no role in the study design or interpretation of the finding.

\section{ACKNOWLEDGMENTS}

We would like to thank Mr. Ming-Kang Li and the Magnetic Resonance Imaging Center of Chang Gung Memorial Hospital for their kind support in technical assistance.

\section{SUPPLEMENTARY MATERIAL}

The Supplementary Material for this article can be found online at: https://www.frontiersin.org/articles/10.3389/fmed. 2021.734410/full\#supplementary-material voxelwise diffusion tensor imaging study. Am J Neuroradiol. (2013) 34:194551. doi: 10.3174/ajnr.A3511

10. Biswal BB, Mennes M, Zuo XN, Gohel S, Kelly C, Smith SM, et al. Toward discovery science of human brain function. Proc Natl Acad Sci USA. (2010) 107:4734-9. doi: 10.1073/pnas.0911855107

11. Liang X, Wen J, Ni L, Zhong J, Qi R, Zhang LJ, et al. Altered pattern of spontaneous brain activity in the patients with end-stage renal disease: a resting-state functional MRI study with regional homogeneity analysis. PLoS ONE. (2013) 8:e71507. doi: 10.1371/journal.pone.0071507

12. Ni L, Wen J, Zhang LJ, Zhu T, Qi R, Xu Q, et al. Aberrant defaultmode functional connectivity in patients with end-stage renal disease: a resting-state functional MR imaging study. Radiology. (2014) 271:54352. doi: 10.1148/radiol.13130816

13. Zheng G, Wen J, Zhang L, Zhong J, Liang X, Ke W, et al. Altered brain functional connectivity in hemodialysis patients with end-stage renal disease: a resting-state functional MR imaging study. Metab Brain Dis. (2014) 29:77786. doi: 10.1007/s11011-014-9568-6

14. Chen HJ Qi R, Kong X, Wen J, Liang X, Zhang Z, et al. The impact of hemodialysis on cognitive dysfunction in patients with end-stage renal disease: a resting-state functional MRI study. Metab Brain Dis. (2015) 30:1247-56. doi: 10.1007/s11011-015-9702-0

15. Shi Y, Tong C, Zhang M, Gao X. Altered functional connectivity density in the brains of hemodialysis end-stage renal disease patients: An in vivo resting-state functional MRI study. PLoS ONE. (2019) 14:e0227123-e. doi: 10.1371 /journal.pone.0227123

16. Jin $\mathrm{M}$, Wang L, Wang $\mathrm{H}$, Han $\mathrm{X}$, Diao Z, Guo W, et al. Structural and functional alterations in hemodialysis patients: a voxel-based morphometry and functional connectivity study. Front Hum Neurosci. (2020) 14:80. doi: 10.3389/fnhum.2020.00080

17. Burton JO, Jefferies HJ, Selby NM, McIntyre CW. Hemodialysis-induced cardiac injury: determinants and associated outcomes. Clin J Am Soc Nephrol. (2009) 4:914-20. doi: 10.2215/CJN.03900808

18. McIntyre CW. Haemodialysis-induced myocardial stunning in chronic kidney disease - a new aspect of cardiovascular disease. Blood Purif. (2010) 29:105-10. doi: 10.1159/000245634 
19. McIntyre CW, Goldsmith DJ. Ischemic brain injury in hemodialysis patients: which is more dangerous, hypertension or intradialytic hypotension? Kidney Int. (2015) 87:1109-15. doi: 10.1038/ki.2015.62

20. Eldehni MT, McIntyre CW. Are there neurological consequences of recurrent intradialytic hypotension? Semin Dial. (2012) 25:253-6. doi: 10.1111/j.1525-139X.2012.01057.x

21. Luo S, Qi RF, Wen JQ, Zhong JH, Kong X, Liang X, et al. Abnormal intrinsic brain activity patterns in patients with end-stage renal disease undergoing peritoneal dialysis: a resting-state functional MR imaging study. Radiology. (2016) 278:181-9. doi: 10.1148/radiol.2015141913

22. Park BS, Seong M, Ko J, Park SH, Kim YW, Hwan Kim I, et al. Differences of connectivity between ESRD patients with PD and HD. Brain Behav. (2020) 10:e01708. doi: 10.1002/brb3.1708

23. Bassett DS, Sporns O. Network neuroscience. Nat Neurosci. (2017) 20:35364. doi: 10.1038/nn.4502

24. Lopez MN, Charter RA, Mostafavi B, Nibut LP, Smith WE. Psychometric properties of the folstein mini-mental state examination. Assessment. (2005) 12:137-44. doi: 10.1177/1073191105275412

25. Kroenke K, Spitzer RL, Williams JB. The Patient Health Questionnaire2: validity of a two-item depression screener. Med Care. (2003) 41:128492. doi: 10.1097/01.MLR.0000093487.78664.3C

26. Song XW, Dong ZY, Long XY Li SF, Zuo XN, Zhu CZ, et al. REST: a toolkit for resting-state functional magnetic resonance imaging data processing. PLoS ONE. (2011) 6:e25031. doi: 10.1371/journal.pone.0025031

27. Wang J, Zuo X, He Y. Graph-based network analysis of resting-state functional MRI. Front Syst Neurosci. (2010) 4:16. doi: $10.3389 /$ fnsys. 2010.00016

28. Tzourio-Mazoyer N, Landeau B, Papathanassiou D, Crivello F, Etard O, Delcroix N, et al. Automated anatomical labeling of activations in SPM using a macroscopic anatomical parcellation of the MNI MRI single-subject brain. Neuroimage. (2002) 15:273-89. doi: 10.1006/nimg.2001.0978

29. Braun U, Plichta MM, Esslinger C, Sauer C, Haddad L, Grimm O, et al. Test-retest reliability of resting-state connectivity network characteristics using fMRI and graph theoretical measures. Neuroimage. (2012) 59:140412. doi: 10.1016/j.neuroimage.2011.08.044

30. Rubinov M, Sporns O. Complex network measures of brain connectivity: uses and interpretations. Neuroimage (2010) 52:1059-69. doi: 10.1016/j.neuroimage.2009.10.003

31. Van Dijk KR, Hedden T, Venkataraman A, Evans KC, Lazar SW, Buckner RL. Intrinsic functional connectivity as a tool for human connectomics: theory, properties, and optimization. J Neurophysiol. (2010) 103:297321. doi: 10.1152/jn.00783.2009

32. Cox RW. AFNI software for analysis and visualization of functional magnetic resonance neuroimages. Comput Biomed Res. (1996) 29:16273. doi: 10.1006/cbmr.1996.0014

33. Son SJ, Kim J, Lee E, Park JY, Namkoong $\mathrm{K}$, Hong $\mathrm{CH}$, et al. Effect of hypertension on the resting-state functional connectivity in patients with Alzheimer's disease (AD). Arch Gerontol Geriatr. (2015) 60:2106. doi: 10.1016/j.archger.2014.09.012

34. Cui Y, Jiao Y, Chen YC, Wang K, Gao B, Wen S, et al. Altered spontaneous brain activity in type 2 diabetes: a resting-state functional MRI study. Diabetes. (2014) 63:749-60. doi: 10.2337/db13-0519

35. Haag S, Friedrich B, Peter A, Häring H-U, Heyne N, Artunc F. Systemic haemodynamics in haemodialysis: intradialytic changes and prognostic significance. Nephrol Dial Transplant. (2018) 33:1419-27. doi: 10.1093/ndt/gfy041

36. Kivimaki I, Lebichot B, Saramaki J, Saerens M. Two betweenness centrality measures based on Randomized Shortest Paths. Sci Rep. (2016) 6:19668. doi: 10.1038/srep19668

37. Biswal B, Yetkin FZ, Haughton VM, Hyde JS. Functional connectivity in the motor cortex of resting human brain using echo-planar MRI. Magn Reson Med. (1995) 34:537-41. doi: 10.1002/mrm.1910340409

38. Christoff K, Irving ZC, Fox KCR, Spreng RN, Andrews-Hanna JR. Mindwandering as spontaneous thought: a dynamic framework. Nat Rev Neurosci. (2016) 17:718-31. doi: 10.1038/nrn.2016.113

39. Huang K-L, Chang T-Y, Ho M-Y, Chen W-H, Yeh M-Y, Chang Y-J, et al. The correlation of asymmetrical functional connectivity with cognition and reperfusion in carotid stenosis patients. NeuroImage Clin. (2018) 20:47684. doi: 10.1016/j.nicl.2018.08.011

40. Tsai PJ, Chen SC, Hsu CY, Wu CW, Wu YC, Hung CS, et al. Local awakening: regional reorganizations of brain oscillations after sleep. NeuroImage. (2014) 102:894-903. doi: 10.1016/j.neuroimage.2014.07.032

41. Fair DA, Cohen AL, Dosenbach NU, Church JA, Miezin FM, Barch DM, et al. The maturing architecture of the brain's default network. Proc Natl Acad Sci USA. (2008) 105:4028-32. doi: 10.1073/pnas.0800376105

42. Gusnard DA, Akbudak E, Shulman GL, Raichle ME. Medial prefrontal cortex and self-referential mental activity: relation to a default mode of brain function. Proc Natl Acad Sci USA. (2001) 98:4259-64. doi: 10.1073/pnas.071043098

43. Utevsky AV, Smith DV, Huettel SA. Precuneus is a functional core of the default-mode network. J Neurosci. (2014) 34:93240. doi: 10.1523/JNEUROSCI.4227-13.2014

44. Ma X, Tian J, Wu Z, Zong X, Dong J, Zhan W, et al. Spatial disassociation of disrupted functional connectivity for the default mode network in patients with end-stage renal disease. PLoS ONE. (2016) 11:e0161392. doi: 10.1371/journal.pone.0161392

45. Stevens FL. Hurley RA, Taber KH. Anterior cingulate cortex: unique role in cognition and emotion. J Neuropsychiatr Clin Neurosci. (2011) 23:1215. doi: 10.1176/jnp.23.2.jnp121

46. Coppola G, Di Renzo A, Tinelli E, Di Lorenzo C, Scapeccia M, Parisi $\mathrm{V}$, et al. Resting state connectivity between default mode network and insula encodes acute migraine headache. Cephalalgia. (2018) 38:84654. doi: $10.1177 / 0333102417715230$

47. Usui C, Kirino E, Tanaka S, Inami R, Nishioka K, Hatta K, et al. Music intervention reduces persistent fibromyalgia pain and alters functional connectivity between the insula and default mode network. Pain Med. (2020) 21:1546-52. doi: 10.1093/pm/pnaa071

48. Santoro D, Satta E, Messina S, Costantino G, Savica V, Bellinghieri G. Pain in end-stage renal disease: a frequent and neglected clinical problem. Clin Nephrol. (2013) 79 Suppl 1:S2-11. doi: 10.5414/CNX77S104

49. Li S, Ma X, Huang R, Li M, Tian J, Wen H, et al. Abnormal degree centrality in neurologically asymptomatic patients with end-stage renal disease: a resting-state fMRI study. Clin Neurophysiol. (2016) 127:6029. doi: 10.1016/j.clinph.2015.06.022

50. Wu CW, Lin S-HN, Hsu L-M, Yeh S-C, Guu S-F, Lee S-H, et al. Synchrony between default-mode and sensorimotor networks facilitates motor function in stroke rehabilitation: a pilot fMRI study. Front Neurosci. (2020) 14:548. doi: 10.3389/fnins.2020.00548

51. Chen HJ, Wen J, Qi R, Zhong J, Schoepf UJ, Varga-Szemes A, et al. Re-establishing brain networks in patients with ESRD after successful kidney transplantation. Clin J Am Soc Nephrol. (2017) 2017:CJN.00420117. doi: 10.2215/CJN.00420117

52. Blumenfeld $H$. Chapter 1 - neuroanatomical basis of consciousness. In: Laureys S, Gosseries O, Tononi G, editors, The Neurology of Conciousness. 2nd ed. San Diego, CA: Academic Press (2016). p. 329. doi: 10.1016/B978-0-12-800948-2.00001-7

53. Chenji S, Jha S, Lee $\mathrm{D}$, Brown $\mathrm{M}$, Seres $\mathrm{P}$, Mah $\mathrm{D}$, et al. Investigating default mode and sensorimotor network connectivity in amyotrophic lateral sclerosis. PLoS ONE. (2016) 11:e0157443. doi: 10.1371/journal.pone.01 57443

54. Schleifer C, Lin A, Kushan L, Ji JL, Yang G, Bearden CE, et al. Dissociable disruptions in thalamic and hippocampal resting-state functional connectivity in youth with 22q112 deletions. J Neurosci Off J Soc Neurosci. (2019) 39:130119. doi: 10.1523/JNEUROSCI.3470-17.2018

55. Cai S, Chong T, Peng Y, Shen W, Li J, von Deneen KM, et al Altered functional brain networks in amnestic mild cognitive impairment: a resting-state fMRI study. Brain Imaging Behav. (2017) 11:619-31. doi: 10.1007/s11682-016-9539-0

56. Ellmore TM, Suescun J, Castriotta RJ, Schiess MCA. Study of the relationship between uric acid and substantia nigra brain connectivity in patients with REM sleep behavior disorder and Parkinson's disease. Front Neurol. (2020) 11:815. doi: 10.3389/fneur.2020.00815

57. Ward PGD, Orchard ER, Oldham S, Arnatkevičiute A, Sforazzini F, Fornito $\mathrm{A}$, et al. Individual differences in haemoglobin concentration influence bold 
fMRI functional connectivity and its correlation with cognition. Neuroimage. (2020) 221:117196. doi: 10.1016/j.neuroimage.2020.117196

Conflict of Interest: The authors declare that the research was conducted in the absence of any commercial or financial relationships that could be construed as a potential conflict of interest.

Publisher's Note: All claims expressed in this article are solely those of the authors and do not necessarily represent those of their affiliated organizations, or those of the publisher, the editors and the reviewers. Any product that may be evaluated in this article, or claim that may be made by its manufacturer, is not guaranteed or endorsed by the publisher.

Copyright (c) 2021 Chang, Wu, Li, Liu, Yeh, Jian, Huang, Lee, Tian and Wu. This is an open-access article distributed under the terms of the Creative Commons Attribution License (CC BY). The use, distribution or reproduction in other forums is permitted, provided the original author(s) and the copyright owner(s) are credited and that the original publication in this journal is cited, in accordance with accepted academic practice. No use, distribution or reproduction is permitted which does not comply with these terms. 